\title{
IWP2 impairs the development of porcine somatic cell nuclear transfer embryos via Wnt signaling pathway inactivation
}

\author{
YONGYE HUANG ${ }^{1,2^{*}}$, LIN YUAN $^{2 *}$, TIANYE LI $^{1}$, XIANGFU LIU ${ }^{1}$, \\ YANG YU ${ }^{1}$, HONGSHENG OUYANG ${ }^{2}$ and BING WANG ${ }^{1}$ \\ ${ }^{1}$ Department of Biochemistry and Molecular Biology, College of Life and Health Sciences, Northeastern University, \\ Shenyang, Liaoning 110169; ${ }^{2}$ Jilin Provincial Key Laboratory of Animal Embryo Engineering, \\ College of Animal Sciences, Jilin University, Changchun, Jilin 130062, P.R. China
}

Received March 14, 2017; Accepted May 11, 2017

DOI: $10.3892 /$ br.2017.918

\begin{abstract}
Wnt signaling is critical in embryonic development and post-embryonic tissue homeostasis. The aim of the present study was to evaluate the expression levels of canonical Wnt signaling genes in porcine somatic cell nuclear transfer (SCNT) embryos. Quantitative polymerase chain reaction analysis was performed in porcine SCNT embryos, and the results indicated that the temporal expression patterns of canonical signaling genes were similar between in vivo and SCNT embryos from the 2-cell to the blastocyst stage. In addition, aberrant expression in a small number of Wnt signaling genes in SCNT embryos was identified. IWP2, an inhibitor of Wnt processing, was applied to the culture of SCNT embryos. The Wnt signaling pathway in the SCNT blastocysts may be inactivated via IWP2 treatment, reflecting the low expression levels of c-Myc and peroxisome proliferator-activated receptor $\delta$. Furthermore, blastocyst hatching was damaged by IWP2 treatment. These findings indicate that the canonical Wnt
\end{abstract}

Correspondence to: Professor Hongsheng Ouyang, Jilin Provincial Key Laboratory of Animal Embryo Engineering, College of Animal Sciences, Jilin University, 5333 Xi'an Road, Changchun, Jilin 130062, P.R. China

E-mail: ouyh@jlu.edu.cn

Professor Bing Wang, Department of Biochemistry and Molecular Biology, College of Life and Health Sciences, Northeastern University, 195 Chuangxin Road, Shenyang, Liaoning 110169, P.R. China

E-mail: wangbing@mail.neu.edu.cn

${ }^{*}$ Contributed equally

Abbreviations: SCNT, somatic cell nuclear transfer; Wnt3A, wingless-type MMTV integration site family, member 3A; Fz3, frizzled homolog 3; Fz4, frizzled homolog 4; APC, adenomatous polyposis coli; Axin, axis inhibition protein; GSK3, glycogen synthase kinase 3; LEF/TCF, lymphoid enhancer-binding factor/T-cell factor

Key words: somatic cell nuclear transfer, gene expression, embryo, Wnt signaling, IWP2 signaling pathway is important for SCNT embryo development.

\section{Introduction}

Somatic cell nuclear transfer (SCNT) is an effective method of reprogramming differentiated nuclei and has many potential applications (1). Owing to incomplete or faulty reprogramming, however, the cloning efficiency of SCNT embryos remains low $(2,3)$. Aberrant reprogramming leads to changes in the expression levels of certain genes important for embryonic development. Studies on the expression of development-associated genes may be valuable for improving the developmental competence of SCNT embryos.

Wnt signaling is a critical regulator of many cellular and physiological processes. Wnt signaling regulates cell proliferation and differentiation, controls migration and patterning during embryonic development, and maintains tissue homeostasis in adults $(4,5)$. Depending on the involvement of $\beta$-catenin, these signaling pathways have been classified as canonical ( $\beta$-catenin-dependent) or non-canonical ( $\beta$-catenin-independent) (6). In the canonical Wnt signaling pathway, which has been better examined (7), $\beta$-catenin accumulates in the cytoplasm and eventually translocates to the nucleus where it acts as a transcriptional co-activator of transcription factors belonging to the T-cell factor/lymphoid enhancer factor-1(TCF/LEF) family. $\beta$-catenin that accumulates in the cytoplasm is degraded, however, by a protein complex that includes axin, glycogen synthase kinase-3 (GSK-3), protein phosphatase 2A (PP2A), casein kinase 1 (CK1) and adenomatous polyposis coli (APC) $(6,8)$. This degradation complex becomes disrupted as soon as the Wnt protein binds to receptors of frizzled $(\mathrm{Fz})$ and low-density lipoprotein receptor-related protein (LRP5/6), which activates multiple signaling cascades.

Dysfunctions in Wnt signaling result in severe complications of embryonic development. For example, abnormalities were observed in the size and shape of mouse embryos lacking intact maternal/zygotic $\beta$-catenin during pre- and post-implantation development (5). By contrast, transient expression of Wnt 2 enhances cellular reprogramming efficiency by elevating $\beta$-catenin nuclear accumulation (9). 
SCNT cloning was associated with altered expression levels of specific Wnt-associated genes in extra-embryonic tissue in a time- and tissue-specific manner in cattle (10). Impaired expression of E-cadherin and $\beta$-catenin proteins, along with defective $\beta$-catenin signaling, contributes to insufficient placentation in bovine SCNT-derived fetuses (11). IWP2 is a potential inhibitor of Wnt processing and secretion; however, studies evaluating the effect of IWP2 on embryonic development are lacking. Therefore, the aim of the present study was to determine whether the low cloning efficiency of SCNT embryos was correlated with irregular coordination between Wnt signaling and embryonic development by applying IWP2 treatment.

\section{Materials and methods}

Somatic cell nuclear transfer. Oocyte collection, in vitro maturation and SCNT were performed in accordance with a previously described protocol (12) with slight modifications. Porcine ovaries were obtained from a local slaughterhouse (HuaZheng Agriculture Development Co. Ltd.) and transported to our laboratory (Jilin Provincial Key Laboratory of Animal Embryo Engineering) within $3 \mathrm{~h}$ in sterile saline solution containing penicillin $(12 \mathrm{mg} / \mathrm{l})$ and streptomycin (20 mg/l) at $30-35^{\circ} \mathrm{C}$. Cumulus-oocyte complexes (COCs) were harvested by aspirating ovarian follicles (diameter, 3-6 mm) with an 18-gauge needle attached to a 10-ml disposable syringe, and COCs with at least three uniform layers of cumulus cells were selected. The selected COCs were rinsed using PVA-TL HEPES stock solution and cultured in TCM-199 (Sigma-Aldrich; Merck KGaA, Darmstadt, Germany) supplemented with $0.1 \%$ polyvinyl alcohol, $3.05 \mathrm{mM}$ D-glucose, $0.91 \mathrm{mM}$ sodium pyruvate, $10 \mathrm{ng} / \mathrm{ml}$ epidermal growth factor (Sigma-Aldrich; Merck KGaA), $0.57 \mathrm{mM}$ cysteine, $0.5 \mu \mathrm{g} / \mathrm{ml}$ follicle-stimulating hormone (Sigma-Aldrich; Merck KGaA), $0.5 \mu \mathrm{g} / \mathrm{ml}$ luteinizing hormone (Sigma-Aldrich; Merck KGaA), $75 \mu \mathrm{g} / \mathrm{ml}$ penicillin and $50 \mu \mathrm{g} / \mathrm{ml}$ streptomycin for $42-44 \mathrm{~h}$ at $38.5^{\circ} \mathrm{C}$ in humidified air containing $5 \% \mathrm{CO}_{2}$.

The cumulus cells of the COCs were denuded by repeated blowing and suction in 0.1\% hyaluronidase with a 100- $\mu l$ pipette. Denuded oocytes with an extruded first polar body, round shape and intact cytoplasm were selected as SCNT recipients. Oocytes were enucleated with a beveled glass pipette by aspirating the first polar body and part of the surrounding cytoplasm. A single donor cell was injected into the perivitelline space of the enucleated oocyte cytoplast with the same glass pipette. SCNT was conducted in manipulation medium (Sigma-Aldrich; Merck KGaA) supplemented with $7.5 \mu \mathrm{g} / \mathrm{ml}$ cytochalasin B (Sigma-Aldrich; Merck KGaA). The oocyte-cell complexes were fused and activated in medium consisting of $0.3 \mathrm{M}$ mannitol, $1.0 \mathrm{mM} \mathrm{MgCl}{ }_{2} \cdot 6 \mathrm{H}_{2} \mathrm{O}, 1.0 \mathrm{mM}$ $\mathrm{CaCl}_{2} \cdot 2 \mathrm{H}_{2} \mathrm{O}$, and $0.5 \mathrm{mM}$ HEPES using a BTX Electro Cell Manipulator 2001 (BTX, San Diego, CA, USA). Reconstructed embryos were then cultured in PZM-3 (12) at $38.5^{\circ} \mathrm{C}$ in a humidified atmosphere containing $5 \% \mathrm{CO}_{2}$. Embryos at the 2-cell, 4-cell, morula, early blastocyst, and hatching blastocyst stages were collected at 24, 36, 96, 132 and $156 \mathrm{~h}$ after activation, respectively.
Collection of in vivo embryos. All animal experiments were conducted according to the guidelines on animal care and use established by the Animal Care and Welfare Committee of Jilin University. All experimental protocols were approved by the Ethics Committee of Jilin University. Gilts for embryo collection were artificially inseminated at estrus and kept in Changchun HuiChang Livestock Co. Ltd. (Changchun, China). Subsequently, 2-cell, 4-cell, morula, and blastocyst embryos were collected by flushing the oviduct or uterus with $10 \%$ fetal bovine serum-phosphate-buffered saline (v/v; Wuhan Boster Biological Technology, Ltd., Wuhan, China) 24, 36, 96, or $132 \mathrm{~h}$ post coitum, respectively. Embryo collection surgery was performed under anesthesia.

Synthesis of single-embryo cDNAs and reverse transcription-quantitative polymerase chain reaction ( $R T-q P C R)$. Briefly, RNA was extracted from 4-5 SCNT or in vivo embryos at the 2-cell, 4-cell, morula, and blastocyst stages. The zona pellucida of each embryo was removed by treatment with Acidic Tyrode's Solution (Sigma-Aldrich; Merck KGaA) and rinsed in bovine serum albumin (Sigma-Aldrich; Merck KGaA)-Dulbecco's phosphate-buffered saline (BSA-DPBS; Gibco; Thermo Fisher Scientific, Inc., Waltham, MA, USA). Each zona-free embryo was immediately selected and transferred into lysis buffer by mouth pipetting. The samples were incubated at $70^{\circ} \mathrm{C}$ for $90 \mathrm{sec}$, followed by $1 \mathrm{~min}$ at $4^{\circ} \mathrm{C}$. Following release of mRNA, SuperScript III reverse transcriptase (Invitrogen; Thermo Fisher Scientfic, Inc.) was used for RT. Exonuclease I (New England BioLabs, Inc., Ipswich, MA, USA) was then used to remove the free primers. A poly (A) tail was added to the 3 '-end of each first-strand cDNA using terminal deoxynucleotidyl transferase (Invitrogen; Thermo Fisher Scientific, Inc.). cDNA amplification was performed with 20 cycles of PCR using TaKaRa Ex Taq ${ }^{\mathrm{TM}}$ HS DNA Polymerase (Takara Bio, Shiga, Japan). Purification of PCR products was conducted with a NucleoSpin ${ }^{\circledR}$ Extract II kit (Macherey-Nagel Co., Düren, Germany).

qPCR was conducted using the $\mathrm{iQ5}^{\mathrm{TM}}$ real-time PCR detection system (Bio-Rad Laboratories, Inc., Hercules, CA, USA). Quantification was performed in triplicate using a BioEasy SYBR-Green I Real Time PCR kit (BIOER, Hangzhou, China) according to the following procedure: Denaturation at $94^{\circ} \mathrm{C}$ for $2 \mathrm{~min}$, followed by 40 cycles of $10 \mathrm{sec}$ at $94^{\circ} \mathrm{C}, 30 \mathrm{sec}$ at the annealing temperature [with primers as previously described (13)], and $30 \mathrm{sec}$ at $72^{\circ} \mathrm{C}$ for extension. The fold change in gene expression was calculated from three replicates with the formula $2^{-\Delta \Delta C q}$ (14) followoing normalization against $\beta$-actin expression.

Inactivation of Wnt signaling. The small-molecule inhibitor IWP2 (Stemgent, Inc., Cambridge, MA, USA) was used to inactivate Wnt signaling in the SCNT embryos. Following electrical activation, embryos were transferred into PZM-3 with 5.0 $\mu \mathrm{M}$ IWP2. Control SCNT embryos were maintained in PZM-3 without any treatment.

Indirect immunofluorescence staining. The zona pellucida of each embryo was removed by treatment with Acidic Tyrode's Solution and rinsed in BSA-DPBS. Zona-free embryos were fixed in $4 \%$ paraformaldehyde $(\mathrm{v} / \mathrm{v})$ for $30 \mathrm{~min}$ at room 

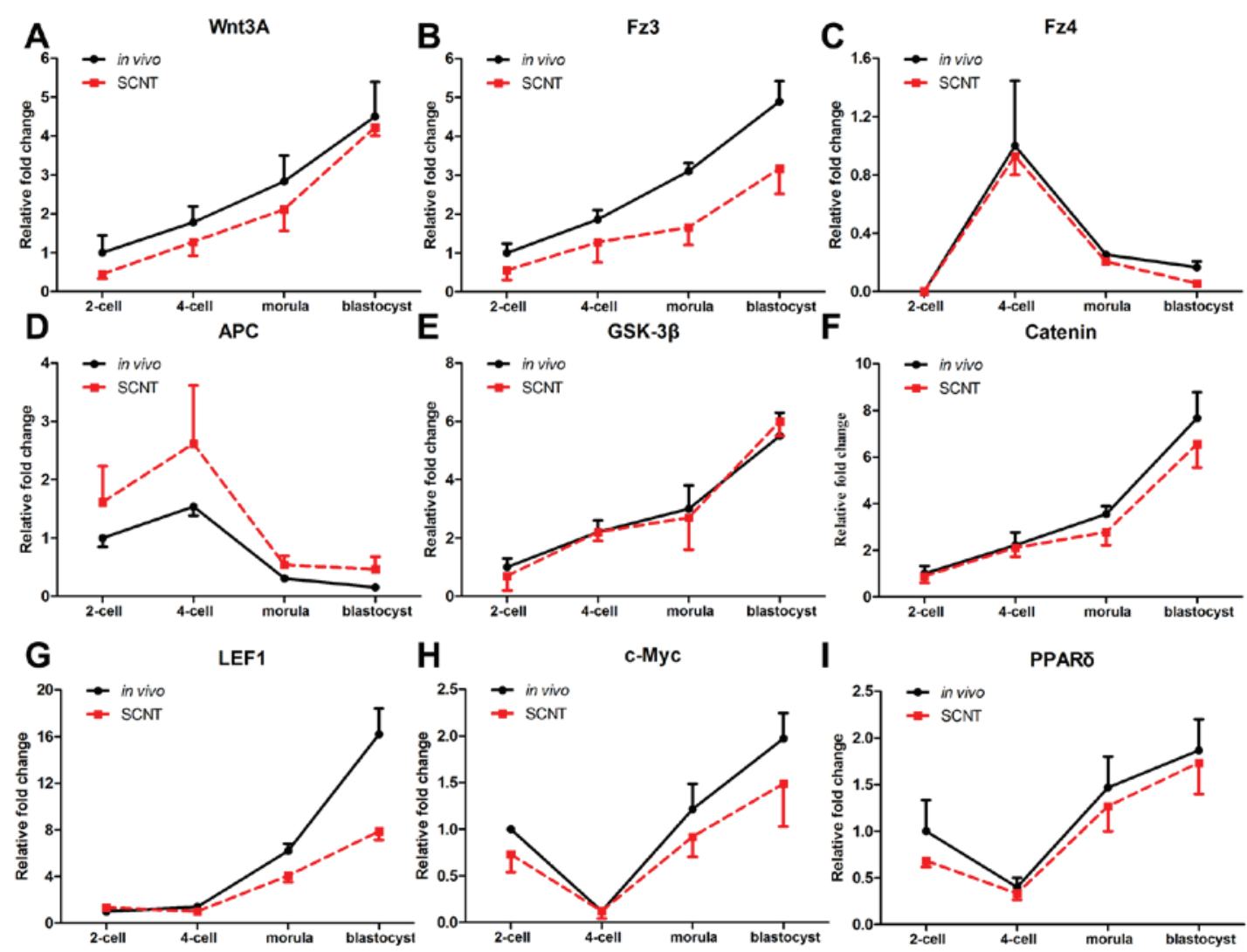

Figure 1. Temporal expression of canonical Wnt signaling components in in vivo and SCNT embryos. The relative fold-change of each gene was calculated following normalization to the expression level of $\beta$-actin in SCNT embryos at the 2-cell stage (Fz4 at the 4-cell stage). SCNT, somatic cell nuclear transfer; Fz4, frizzled homolog 4; Fz3, frizzled homolog 3; APC, adenomatous polyposis coli; GSK, glycogen synthase kinase; LEF1, lymphoid enhancer binding factor 1 ; PPAR $\delta$, peroxisome proliferator activated receptor $\delta$.

temperature and permeabilized with $0.2 \%$ Triton X-100 (Sigma-Aldrich; Merck KGaA) for 30 min. Embryos were blocked in 5\% goat serum (Wuhan Boster Biological Technology, Ltd.) in PBS for 30 min and incubated overnight with primary antibodies at $4^{\circ} \mathrm{C}$. Primary antibodies used in the analysis were anti- $\beta$-catenin mouse monoclonal antibody (1:200; sc-65480; Santa Cruz Biotechnology, Inc., Dallas, TX, USA) and anti-active- $\beta$-catenin mouse monoclonal antibody (1:500; 05-655; Upstate Biotechnology, Inc., Lake Placid, NY, USA). Subsequent to removing the primary antibodies, embryos were rinsed three times with $0.2 \%$ Tween-20 in PBS. Embryos were incubated with Alexa Fluor 594 goat anti mouse $\operatorname{IgG}(1: 2,000$; R37121; Invitrogen; Thermo Fisher Scientific, Inc.) at $4^{\circ} \mathrm{C}$ overnight. Embryos were stained with Hoechst 33342 and observed under an epifluorescent microscope (Nikon Corporation, Tokyo, Japan).

\section{Results}

Temporal expression patterns of Wnt signaling genes in porcine pre-implantation embryos. To determine the roles of canonical Wnt signaling pathway genes in porcine SCNT embryos, their temporal expression patterns were examined. The expression of various canonical Wnt signaling genes, including Wnt3A, frizzled receptor transcripts (Fz3 and Fz4), adenomatous polyposis coli (APC), glycogen synthase kinase (GSK) $3 \beta, \beta$-catenin, lymphoid enhancer binding factor 1 (LEF1), c-Myc and peroxisome proliferator activated receptor
(PPAR) $\delta$, was analyzed in individual porcine embryos by RT-qPCR. As shown in Fig. 1, changes in the expression patterns of Wnt signaling genes were similar between in vivo and SCNT embryos from the 2-cell to the blastocyst stage. The expression level of Wnt3A, Fz3, GSK3 $\beta$, $\beta$-catenin and LEF1 steadily increased from the 2-cell to the blastocyst stage (Fig. 1A,B,E-G). For Fz4 and APC, there was a peak in expression at the 4-cell stage, and expression then gradually declined to the blastocyst stage (Fig. 1C and D). By contrast, the expression levels of Wnt target genes, c-Myc and PPARס decreased from the 2-cell to the 4-cell stage, and subsequently increased, reaching a peak at the blastocyst stage (Fig. 1H and I). Thus, the temporal expression patterns of canonical Wnt signaling genes in SCNT embryos were similar with those of in vivo embryos.

Development of SCNT embryos was compromised with Wnt signaling inactivation. The results revealed that various genes were expressed abnormally in pre-implantation embryos, although the actual contribution of the Wnt signaling pathway to the development competence of SCNT embryos requires further elucidation. Our previous study revealed that parthenogenetic blastocyst hatching may be affected by activation or inactivation of Wnt signaling. Therefore, the effects of Wnt signaling blockade were accessed in SCNT embryos. As presented in Table I, no significant differences were identified between control and IWP2-treated embryos in blastocyst-formation rates or cell numbers in the early 
Table I. Effects of IWP2 on the in vitro blastocyst development of somatic cell nuclear transfer embryos.

\begin{tabular}{lcccc}
\hline Treatment & $\begin{array}{c}\text { Total } \\
\text { oocytes }\end{array}$ & $\begin{array}{c}\text { Early blastocysts } \\
(\% \pm \text { SD })\end{array}$ & $\begin{array}{c}\text { Hatching blastocysts } \\
{[\% \pm S D)}\end{array}$ & $\begin{array}{c}\text { Cell numbers in early blastocysts } \\
(\text { mean } \pm \text { SD })\end{array}$ \\
\hline Control & 948 & $248(28.3 \pm 9.1)^{\mathrm{a}}$ & $153(64.3 \pm 11.1)^{\mathrm{a}}$ & $46 \pm 10.2^{\mathrm{a}}$ \\
IWP2 & 575 & $160(27.4 \pm 8.8)^{\mathrm{a}}$ & $25(15.4 \pm 2.2)^{\mathrm{b}}$ & $47 \pm 8.1^{\mathrm{a}}$ \\
\hline
\end{tabular}

${ }^{\mathrm{a}, \mathrm{b}}$ Values with different superscripts within the same column denote significant difference $(\mathrm{P}<0.05)$. The blastocyst-formation rate was calculated by dividing the number of early blastocysts to the number of oocytes. The blastocyst hatching rate was based on the number of hatching blastocysts divided by the number of early blastocysts.
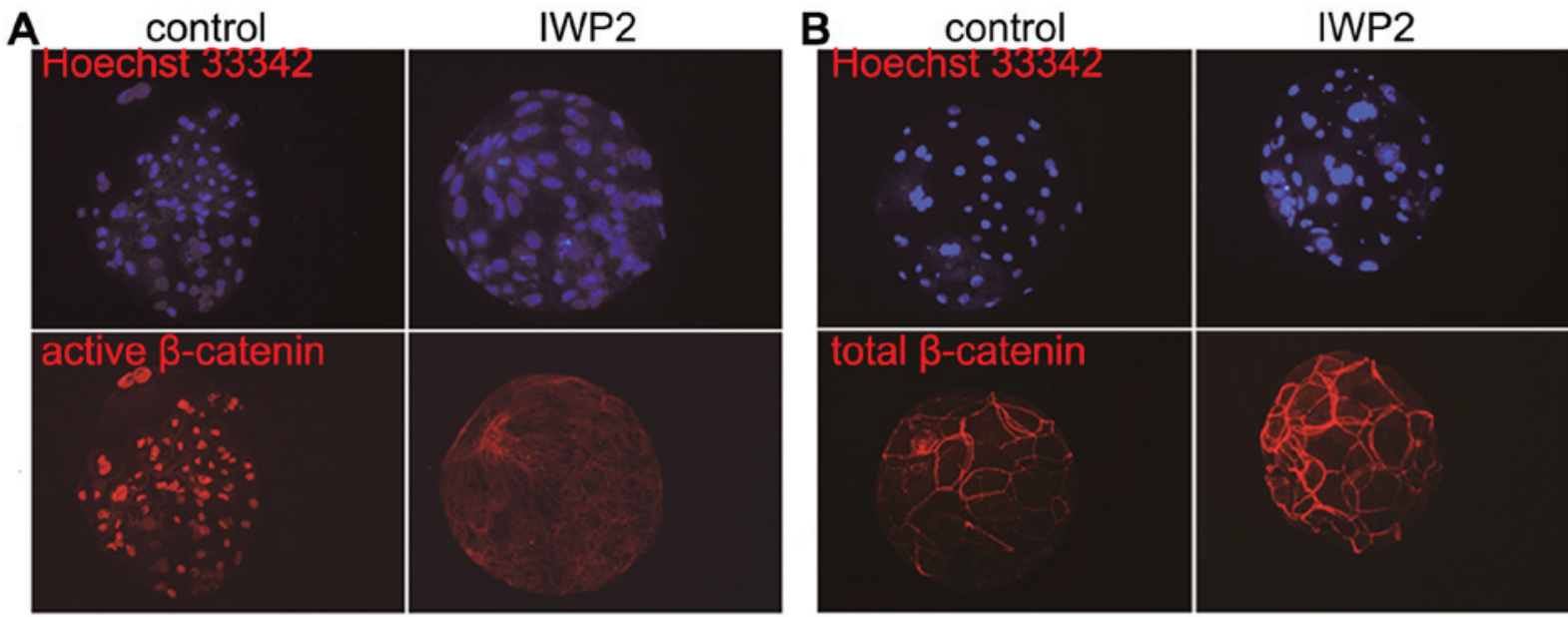

Figure 2. Expression of (A) active and (B) total $\beta$-catenin in porcine somatic cell nuclear transfer blastocysts treated with or without IWP-2 as determined by immunofluorescence staining. Magnification, x300.
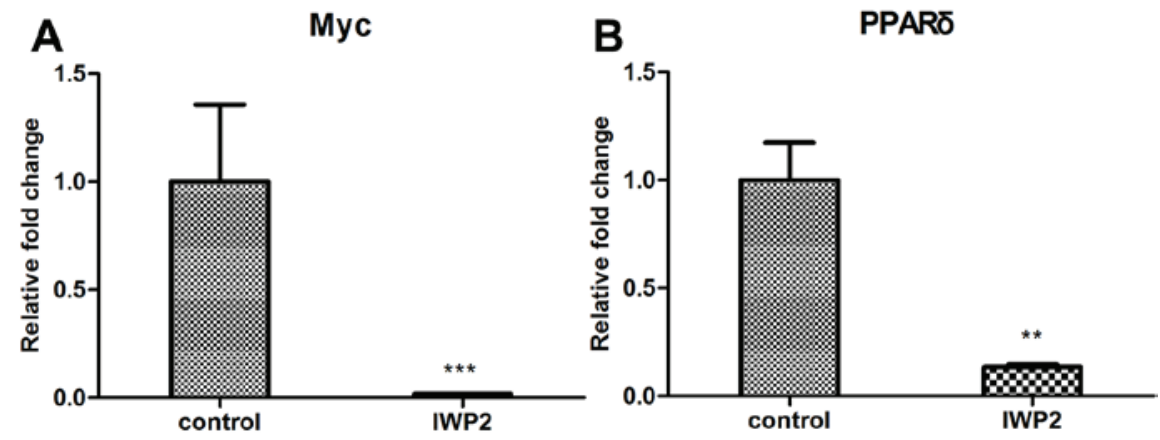

Figure 3. Gene expression in porcine SCNT blastocysts treated with or without IWP2. The relative fold change of each gene was calculated following normalization to its level in blastocysts without IWP2 treatment. The data are presented as means \pm standard deviation, $\mathrm{n}=3{ }^{, * *} \mathrm{P}<0.01 \mathrm{vs}$. control, and ${ }^{* * * *} \mathrm{P}<0.001$ vs. control. SCNT, somatic cell nuclear transfer.

blastocyst. However, the hatching rate of embryos at $156 \mathrm{~h}$ was significantly reduced following IWP2 treatment.

To confirm the inhibition of Wnt signaling following IWP2 exposure, $\beta$-catenin immunofluorescence was observed in SCNT blastocysts as cytosolic $\beta$-catenin is transported into the nucleus when Wnt signaling is active. As shown in Fig. 2, no significant differences in total $\beta$-catenin abundance between control and IWP2-treated blastocysts were observed, although expression of active $\beta$-catenin was nearly undetectable following IWP2 treatment. In addition, expression levels of the Wnt target genes, c-Myc and PPARS were analyzed. As presented in Fig. 3A and B, expression levels of c-Myc and PPARd were downregulated in SCNT blastocyst following IWP2 treatment. These results confirm that the Wnt signaling pathway is inactivated by IWP2 treatment.

\section{Discussion}

The Wnt signaling pathway is important for embryonic development, tissue regeneration and homeostasis $(15,16)$. In the present study, certain Wnt signaling genes were observed to be aberrantly expressed in SCNT embryo, such as the expression of Wnt3A, FZ3, LEF1 and APC. In our previous study, it 
was primarily Wnt genes that exhibited different expression patterns in parthenogenetic and in vivo embryos (13). This may be one of the reasons for differences in developmental competence between parthenogenetic and SCNT embryos. While parthenogenetic embryos do not develop to term, a small number of SCNT embryos go through gestation and form cloned piglets.

Furthermore, the present study identified that blastocyst hatching was impaired following IWP2 treatment to inactivate the Wnt signaling pathway. Furthermore, $\beta$-catenin has been shown to be aberrantly expressed in bovine SCNT placentas (11). These findings indicate that Wnt signaling is markedly more important for trophectoderm development, affecting SCNT embryonic development by disturbing placentation. Notably, one component of Wnt signaling, LEF1, which was downregulated in SCNT embryos at the morula and blastocyst stages in the present study, is critical in the development of the placenta (17). Such aberrant gene expression patterns may contribute to the failure of embryonic development or to defects in the SCNT piglets. However, the present study did not identify the most critical Wnt gene for embryonic development. Further research should be focused on regulating the expression of Wnt signaling genes to improve SCNT reprogramming efficiency and embryonic development.

In summary, the present study demonstrated that only a small proportion of Wnt signaling genes were abnormally expressed during pre-implantation development, and this pathway serves important roles in the development of the trophectoderm in porcine SCNT blastocysts.

\section{Acknowledgements}

The authors would like to thank Mr. Wanhua Xie, Miss Xianju Chen, Mr. Yang Han, Professor Xiaochun Tang, Professor Daxin Pang and Professor Zhanjun Li for technical support and helpful discussion. This work was supported by the China National Key Basic Research Program (grant no. 2011CB944200), the National Natural Science Foundation of China (grant no. 81502582), the Fundamental Research Funds of Northeastern University (grant nos. N152004003 and N141008001-8), and the Postdoctoral Scientific Research Funds of Northeastern University (grant no. 20150314).

\section{References}

1. Iuso D, Czernik M, Zacchini F, Ptak G and Loi P: A simplified approach for oocyte enucleation in mammalian cloning. Cell Reprogram 15: 490-494, 2013.
2. Thuan NV, Kishigami S and Wakayama T: How to improve the success rate of mouse cloning technology. J Reprod Dev 56: 20-30, 2010.

3. Huang Y, Ouyang H, Yu H, Lai L, Pang D and Li Z: Efficiency of porcine somatic cell nuclear transfer - a retrospective study of factors related to embryo recipient and embryos transferred. Biol Open 2: 1223-1228, 2013.

4. MacDonald BT, Tamai K and He X: Wnt/beta-catenin signaling: components, mechanisms, and diseases. Dev Cell 17: 9-26, 2009.

5. Messerschmidt D, de Vries WN, Lorthongpanich C, Balu S, Solter D and Knowles BB: beta-catenin-mediated adhesion is required for successful preimplantation mouse embryo development. Development 143: 1993-1999, 2016.

6. Bengoa-Vergniory N and Kypta RM: Canonical and noncanonical Wnt signaling in neural stem/progenitor cells. Cell Mol Life Sci 72: 4157-4172, 2015.

7. Fujimaki S, Wakabayashi T, Takemasa T, Asashima M and Kuwabara T: The regulation of stem cell aging by Wnt signaling. Histol Histopathol: 11657, 2015.

8. Minde DP, Anvarian Z, Rudiger SGD and Maurice MM: Messing up disorder: how do missense mutations in the tumor suppressor protein APC lead to cancer? Mol Cancer 10: 101, 2011.

9. Kimura M, Nakajima-Koyama M, Lee J and Nishida E: Transient Expression of WNT2 Promotes Somatic Cell Reprogramming by Inducing beta-Catenin Nuclear Accumulation. Stem Cell Reports 6: 834-843, 2016.

10. Biase FH, Rabel C, Guillomot M, et al: Changes in WNT signaling-related gene expression associated with development and cloning in bovine extra-embryonic and endometrial tissues during the peri-implantation period. Mol Reprod Dev 80: 977-987, 2013.

11. Kohan-Ghadr HR, Smith LC, Arnold DR, Murphy BD and Lefebvre RC: Aberrant expression of E-cadherin and beta-catenin proteins in placenta of bovine embryos derived from somatic cell nuclear transfer. Reprod Fertil Dev 24: 588-598, 2012.

12. Lai L, Kolber-Simonds D, Park KW, et al: Production of alpha-1,3-galactosyltransferase knockout pigs by nuclear transfer cloning. Science 295: 1089-1092, 2002.

13. Huang Y, Ouyang H, Xie W, et al: Moderate expression of Wnt signaling genes is essential for porcine parthenogenetic embryo development. Cellular signalling 25: 778-785, 2013.

14. Livak KJ and Schmittgen TD: Analysis of relative gene expression data using real-time quantitative PCR and the $2(\mathrm{~T})$ (-Delta Delta C) method. Methods 25: 402-408, 2001.

15. Caprioli A, Villasenor A, Wylie LA, Braitsch C, Marty-Santos L, Barry D, Karner CM, Fu S, Meadows SM, Carroll TJ and Cleaver O: Wnt4 is essential to normal mammalian lung development. Dev Biol 406: 222-234, 2015.

16. Vlad-Fiegen A, Langerak A, Eberth S and Muller O: The Wnt pathway destabilizes adherens junctions and promotes cell migration via beta-catenin and its target gene cyclin D1. FEBS Open Bio 2: 26-31, 2012.

17. Galceran J, Farinas I, Depew MJ, Clevers H and Grosschedl R: Wnt3a(-/-)-like phenotype and limb deficiency in Lef1(-/-) Tcf1(-/-) mice. Genes \& Development 13: 709-717, 1999. 